\title{
MiR-10a-5p: A Promising Biomarker for Early Diagnosis and Prognosis Evaluation of Bladder Cancer
}

\author{
Lei Yang \\ Hong-Fang Sun \\ Lin-Qing Guo \\ Hai-Bing Cao
}

Department of Urology, Wuxi No.2 Chinese Medicine Hospital, Wuxi City, Jiangsu Province, 214I2I, People's Republic of China
Correspondence: Lei Yang Department of Urology, Wuxi No.2 Chinese Medicine Hospital, No. 390 Xincheng Road, Taihu Street, Binhu District, Wuxi City, Jiangsu Province, 2I4I2I, People's Republic of China Email leiyang_0312@I63.com
Introduction: MiRNAs play a critical role in carcinogenesis, among which miR-10a-5p has been reported in several types of human cancer. Nevertheless, the role of miR-10a-5p remain uncovered in bladder cancer $(\mathrm{BCa})$.

Methods: We recruited $88 \mathrm{BCa}$ patients and 36 healthy controls (HC) to form the training cohort, and other $120 \mathrm{BCa}$ patients to form the validation cohort. The clinical samples were collected for analysis. The expression level of miR-10a-5p was evaluated using RT-qPCR. Receiver operating characteristic (ROC) curves were utilized to calculate diagnostic accuracy. Survival curves were generated to analyze survival outcomes. CCK-8 and transwell assays were conducted to test the cell proliferation, migration, and invasion capacities.

Results: MiR-10a-5p was upregulated in human $\mathrm{BCa}$ tissues and closely associated with advanced clinicopathological features, including advanced tumor grade, histological grade, and T stage. High expression of miR-10a-5p was associated with worse survival outcomes in $\mathrm{BCa}$ patients. Circulating plasma miR-10a-5p expression had the great performance power to discriminate $\mathrm{BCa}$ patients form $\mathrm{HC}$ patients before surgery, and to differentiate muscle invasive bladder cancer (MIBC) from non-muscle invasive bladder cancer (NMIBC). In addition, overexpression of miR-10a-5p could promote $\mathrm{BCa}$ cell proliferation, migration, and invasion.

Conclusion: This study indicates that miR-10a-5p is a crucial diagnostic and prognostic biomarker for BCa patients, and miR-10a-5p exerted a tumor promoting role during $\mathrm{BCa}$ cell progression.

Keywords: bladder cancer, miR-10a-5p, diagnostic, prognosis, biomarker

\section{Introduction}

Bladder cancer $(\mathrm{BCa})$ is one of the most frequently occurring malignancies worldwide, with about 380,000 new cases and 15,000 deaths each year. ${ }^{1}$ The incidence of $\mathrm{BCa}$ in males is three times that in females. ${ }^{2} \mathrm{BCa}$ incidence and mortality rates vary across countries due to differences in risk factors, diagnostic practices, and availability of treatments. ${ }^{3}$ BCa has different histological types including urothelial carcinoma, squamous cell carcinoma and adenocarcinoma, and urothelial carcinoma is the most frequent histological type, accounting for more than $95 \%$ of all $\mathrm{BCa}{ }^{4}$ Recently, despite the obvious progress in diagnosis and treatment, the long-term prognosis remains poor for $\mathrm{BCa}$ patients, with the 5-year overall survival remaining only $50 \%-60 \%$. ${ }^{5}$ The high tumor recurrence rate and a high migratory and invasive ability of BCa have contributed greatly to the unsatisfactory prognosis. ${ }^{6}$ With the increasing understanding of molecular abnormality, molecular diagnosis and targeted therapy 
have developed into a crucial part for the diagnosis and treatment of many cancers. ${ }^{7}$ Moreover, some personalized approaches have grown rapidly for $\mathrm{BCa}$ treatment over the past several years, including novel targeted small-molecule and biological treatments, as well as immunotherapies. ${ }^{8}$ Several immune checkpoint inhibitors that target programmed cell death protein 1 (PD1), its ligand PDL1, and cytotoxic T lymphocyte-associated protein 4 (CTLA4) have already been approved for use in bladder cancer. ${ }^{9}$ Hence, it is urgent to seek effective and efficient biomarkers for the diagnosis and treatment of $\mathrm{BCa}$ patients. ${ }^{10}$

MicroRNAs (miRNAs) are endogenous small noncoding RNAs at a length of 19 to 25 nucleotide. Recently, researches have elucidated the important roles of miRNAs in carcinogenesis, including cell differentiation, proliferation, apoptosis, and cell metastasis. ${ }^{11-13}$ Moreover, miRNAs can act as either oncogene or tumor suppressors depending on the genes it targets in various types of cancers. ${ }^{14}$ Recently, the role of miRNAs has been increasingly determined for the early diagnosis and targeted treatment for tumors of urinary system, including prostate cancer and $\mathrm{BCa}$ patients. ${ }^{15,16} \mathrm{MiR}-10 \mathrm{a}-5 \mathrm{p}$, which has been widely reported in recent years, plays critical roles in different kinds of cancers including pancreatic ductal adenocarcinoma, ${ }^{17}$ acute myeloid leukemia, ${ }^{18}$ ovarian cancer, ${ }^{19}$ lung cancer, ${ }^{20}$ renal cell carcinoma, ${ }^{21}$ and cervical cancer. ${ }^{22}$ Nevertheless, the role of miR-10a-5p still remain uncovered in $\mathrm{BCa}$.

In this study, we determined that miR-10a-5p was upregulated in human $\mathrm{BCa}$ tissues, and its high expression could be an indicator for worse survival. Moreover, circulating plasma miR-10a-5p expression employed outstanding diagnostic value for $\mathrm{BCa}$ patients. In addition, enhanced expression of miR-10a-5p promoted proliferation, migration, and invasion of $\mathrm{BCa}$ cells.

\section{Materials and Methods}

\section{Clinical Samples}

A total of $88 \mathrm{BCa}$ patients and 36 healthy controls (HC) were recruited from the Wuxi No.2 Chinese Medicine Hospital between January 2009 and December 2014 to constitute the training cohort. Meanwhile, other $120 \mathrm{BCa}$ patients were enrolled at the same institution between January 2012 and May 2016 to form the validation cohort. Out of 88 bladder cancer patients in training cohort, 32 were diagnosed with non-muscle invasive bladder cancer (NMIBC); while the other 56 were diagnosed with muscle invasive bladder cancer (MIBC). Out of 120 bladder cancer patients in validation cohort, 40 were diagnosed with NMIBC; while the other 80 were diagnosed with MIBC. In the training cohort, plasma samples were extracted on the day of admission (BCa patients and $\mathrm{HC}$ patients) and 1 month after surgery (BCa patients); while in the validation cohort, plasma samples were extracted from BCa patients on the day of admission. The tumor tissues and paired adjacent normal tissues were obtained from $\mathrm{BCa}$ patients after surgery both in training and validation cohort. The inclusion criteria for $\mathrm{BCa}$ patients were: ${ }^{1}$ all patients were histologically confirmed as $\mathrm{BCa} ;{ }^{2}$ no other associated malignancies; ${ }^{3}$ all patients underwent primary section of bladder cancer (TURBt) or radical cystectomy (RC) without other presurgical anti-cancer treatments; ${ }^{4}$ patients had complete follow-up and clinicopathological information. The peripheral blood samples were collected from all participants in EDTA gel tubes. Each sample was centrifuged at $2000 \mathrm{~g}$ for ten minutes to separate plasma and then stored at $-80^{\circ} \mathrm{C}$ until tested. The collected tumor tissues and paired normal tissues were immediately frozen in liquid nitrogen and frozen at $-80^{\circ} \mathrm{C}$ before tested. Some clinical data were collected including age, gender, tumor grade, histological grade, T stage, lymph nodes metastasis, and multiplicity. Written informed consent was obtained from all the patients. This study was approved by the ethics committee of Wuxi No.2 Chinese Medicine Hospital and was conducted in accordance with the Declaration of Helsinki.

\section{Cell Culture}

Human $\mathrm{BCa}$ cell line $253 \mathrm{j}$ and $\mathrm{J} 82$ was obtained from Shanghai Chinese Academy of Sciences cell bank (China). 253j and J82 cells were cultured in DMEM medium (Life Technologies, Carlsbad, CA, US) supplemented with $10 \%$ fetal bovine serum (FBS, Life Technologies), $100 \mathrm{U} / \mathrm{mL}$ penicillin and streptomycin under a humidified incubator at $37{ }^{\circ} \mathrm{C}$ with $5 \% \mathrm{CO}_{2}$.

\section{RNA Extraction and Quantitative Reverse Transcription-Polymerase Chain Reaction (RT-qPCR)}

Total RNA was extracted from tissues or cells using the RNA Isolation Kit (Qiagen, USA). Complementary DNA (cDNA) was synthesized using the RevertAid First Strand cDNA Synthesis Kit (Thermo Fisher, USA) in accordance with the manufacturer's protocol. RT-qPCR reactions were then conducted on the $\mathrm{ABI}$ prism 7500 sequence detection 
system (Applied Biosystems) using the TaqMan miR assay system (Applied Biosystems, CA, USA). The thermal cycling conditions were: $95^{\circ} \mathrm{C}$ for $20 \mathrm{sec}$, followed by 40 cycles of $94^{\circ} \mathrm{C}$ for $20 \mathrm{sec}, 60^{\circ} \mathrm{C}$ for $40 \mathrm{sec}$ and $72^{\circ} \mathrm{C}$ for 10 sec. Relative expression of miR-10a-5p were normalized to that of $U 6$ using $2^{-\Delta \Delta \mathrm{Ct}}$ method. ${ }^{23}$ The sequences of oligonucleotides (GenePharma Co., Ltd, Shanghai, China) used in this study are as follows: miR-10a-5p: forward: $5^{\prime}-$ CGCTAGAAGCTTTTGGGTTA-3', reverse: 5'-GCCCT AGACCATGGATTT-3'; U6: forward: 5'-CGCTTCG GCAGCACATATAC-3', reverse: 5'-TTCACGAATTTGC GTGTCAT-3'.

\section{Cell Transfection}

BCa cells were transfected with miR-10a-5p mimics, miR10a-5p inhibitors, or their corresponding negative controls (NC mimics or NC inhibitors) (GenePharma Co., Ltd, Shanghai, China). Briefly, BCa cells were seeded in 6 -well plates at a density of $1 \times 10^{5}$ cells per well for $24 \mathrm{~h}$. Cell transfection was conducted using Lipofectamine 2000 (Invitrogen, Carlsbad, CA, USA) based on the manufacturer's instructions. Transfected cells were further cultured for an additional $48 \mathrm{~h}$ at $37^{\circ} \mathrm{C}$ before being used in downstream experiments.

\section{Cell Counting Kit-8 (CCK-8) Assay}

Three replicates of transfected $\mathrm{BCa}$ cells were seeded into 96-well plates at a density of $1 \times 10^{3}$ cells per well, following which $10 \mu \mathrm{L}$ of CCK-8 solution (Dojindo, Kumamoto, Japan) was added to the medium at 1, 2, 3, 4 day before the cells were further incubated at $37{ }^{\circ} \mathrm{C}$ for $2 \mathrm{~h}$. Optical density (OD) values were measured at $450 \mathrm{~nm}$ using an automatic microplate reader (Bio-Rad Laboratories, Inc., Hercules, CA, USA).

\section{Transwell Assay}

The polyethylene membranes (24-well inserts; $8.0 \mu \mathrm{m}$; Corning, Inc.) were utilized to detect the migration and invasion abilities of $\mathrm{BCa}$ cells. Chambers precoated with $50 \mu \mathrm{L}$ Matrigel (BD Biosciences) at $37^{\circ} \mathrm{C}$ for $1 \mathrm{~h}$ were used for invasion assays, while uncoated chambers were utilized for migration assays. Cell suspensions containing $1 \times 10^{5}$ cells in $100 \mu \mathrm{L}$ FBS-free DMEM were seeded in the upper chamber. Meanwhile, the lower chamber was covered with $500 \mu \mathrm{L}$ DMEM supplemented with $10 \%$ FBS Cells were cultured at $5 \% \mathrm{CO}_{2}$ and $37^{\circ} \mathrm{C}$ for 48 hours. After 48 hours, cells that have remained in the upper membranes were gently removed using a cotton swab; while cells that have migrated or invaded the bottom of the membrane were fixed with polyoxymethylene at room temperature for $20 \mathrm{~min}$ and then stained with $0.5 \%$ crystal violet at room temperature for $20 \mathrm{~min}$. Cells were counted in 5 randomly selected fields under a light microscope (Leica Microsystems GmbH, Wetzlar, Germany).

\section{Statistical Analysis}

All data are expressed as mean \pm standard deviation of at least three experiments. Statistical evaluations were performed using SPSS 20.0 (IBM SPSS Inc., Chicago, IL, USA). Differences between two groups were analyzed using unpaired Student's $t$-test, while the expression of miR-10a-5p in tumor tissues and matched normal tissues was compared using paired Student's $t$-test. Comparisons of multiple groups were analyzed using the ANOVA followed by Dunnett's test. Categorical data were compared using chi-square test. Receiver operating characteristic (ROC) curves were utilized to calculate diagnostic accuracy. Overall survival (OS) and recurrence-free (RFS) of patients with $\mathrm{BCa}$ were evaluated using Kaplan-Meier curves and compared using Log rank test. Prognostic factors were analyzed by Cox regression proportional hazards analysis. Differences were considered to be significant when $P<0.05$.

\section{Results}

\section{MiR-I0a-5p is Upregulated in BCa Tissues}

We firstly detected the expression profiles of miR-10a-5p in $\mathrm{BCa}$ tissues in the training cohort, and a total of $88 \mathrm{BCa}$ tissues and paired normal tissues were collected for RT-qPCR analysis. Our results showed that miR-10a-5p expression was significantly upregulated in primary $\mathrm{BCa}$ tissues compared to that in paired normal tissues ( $P<0.001$, Figure 1A). Moreover, RTqPCR analysis showed a significant upregulation of miR-10a$5 \mathrm{p}$ in patient with MIBC compared to those with NMIBC $(P<$ 0.001 , Figure 1B). Subsequently, the relative expression levels of miR-10a-5p were evaluated in plasma samples of the 32 patients with NMIBC, 56 patients with MIBC and $36 \mathrm{HC}$ patients. RT-qPCR analysis results showed that miR-10a-5p expression was significantly higher in the MIBC group compared to the NMIBC or HC group $(P<0.001$, Figure 1C). The plasma levels of miR-10a-5p were also measured by RT-qPCR at 1 month after surgery, and the results showed that plasma levels of miR-10a-5p were significantly downregulated at 1 month after treatment when compared to the pre-surgery levels of miR-10a-5p $(P<0.001$, Figure 1D). We analyzed the 
A
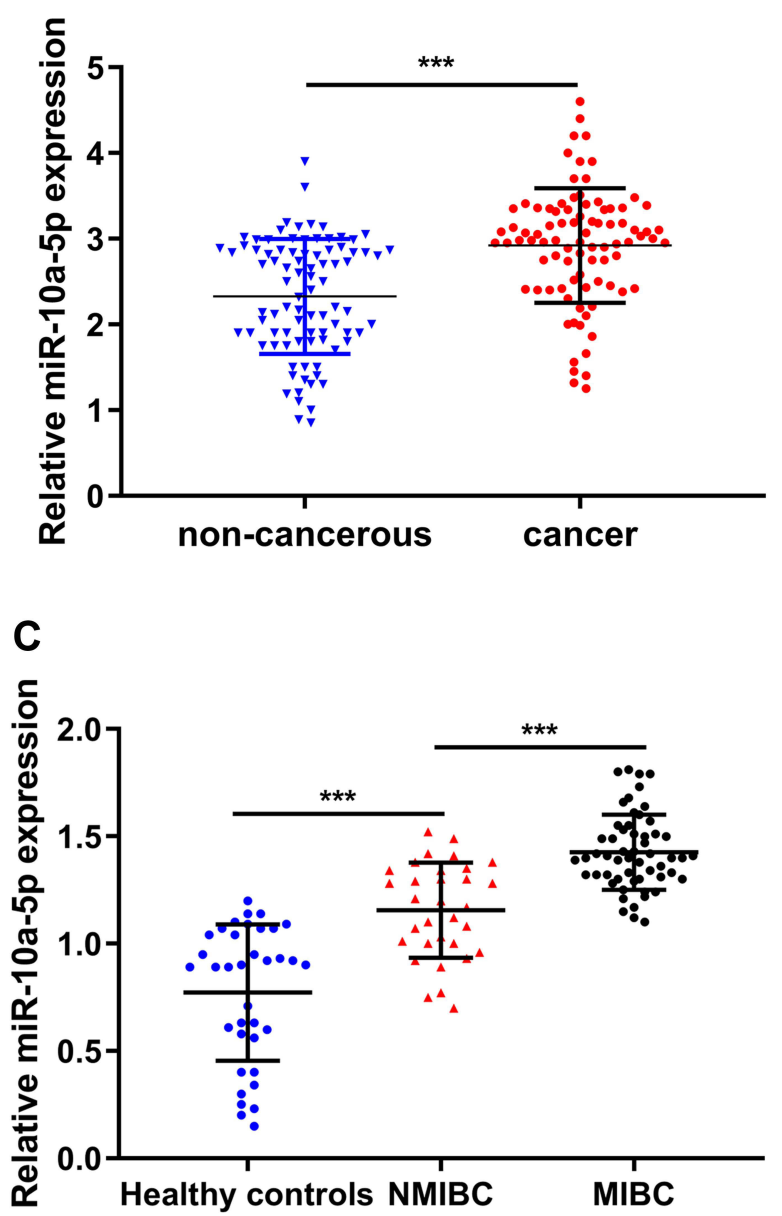

B
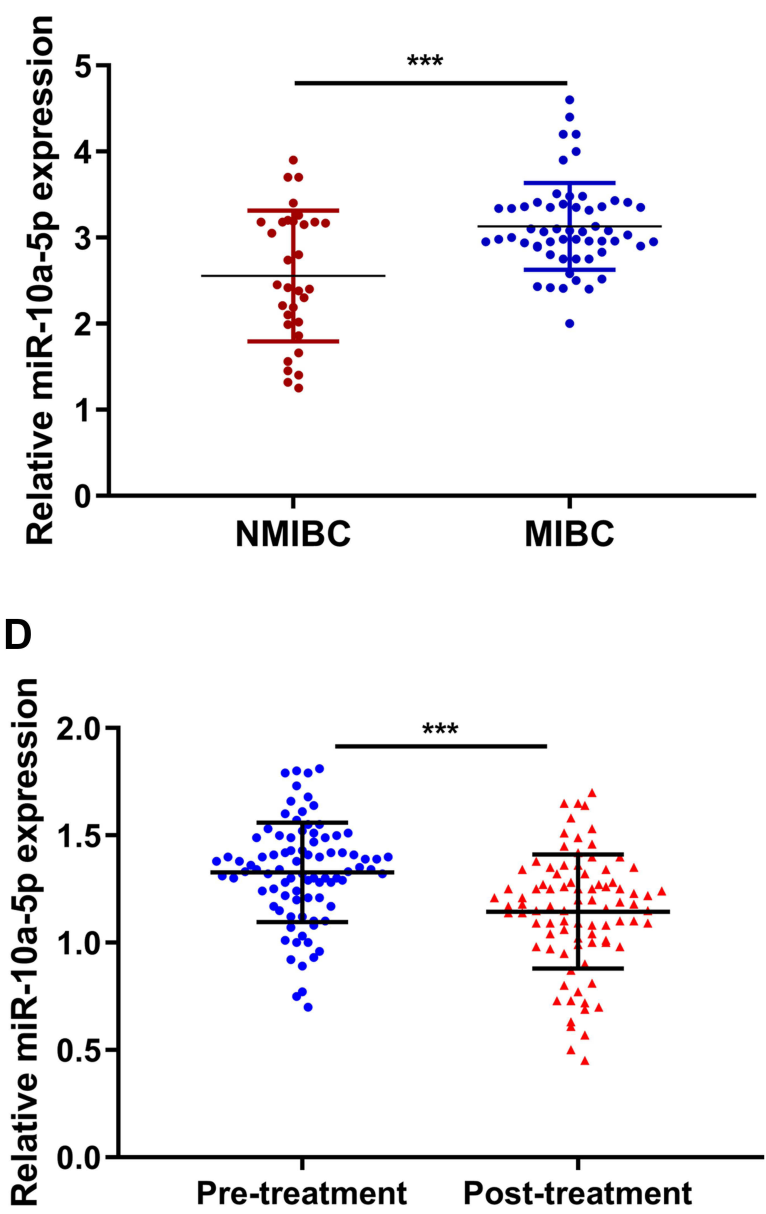

Figure I Upregulation miR-10a-5p in BCa tissues. (A) RT-qPCR analysis of miR-10a-5p expression in 88 pairs of BCa tissues and adjacent normal tissues. (B) RT-qPCR analysis of miR-10a-5p expression in patients with MIBC or NMIBC. (C) RT-qPCR analysis of miR-10a-5p expression in plasma samples of 60 MIBC, NMIBC, and healthy controls (HC) patients. (D) Downregulation of miR-10a-5p was observed after surgical resection. $* * * P<0.00 \mathrm{I}$.

Abbreviations: $\mathrm{BC}$, bladder cancer; MIBC, muscle invasive bladder cancer; NMIBC, non-muscle invasive bladder cancer.

relationship between miR-10a-5p expression in BCa tissues and the clinicopathological features of these BCa patients. We firstly separated the $88 \mathrm{BCa}$ patients into miR-10a-5p low expression group $(\mathrm{n}=44)$ and miR-10a-5p high expression group $(n=44)$ based on the median values of miR-10a-5p expression in $\mathrm{BCa}$ tissues. MiR-10a-5p expression in tumor tissues was significantly correlated with tumor grade, histological grade, and T stage (Table 1). Moreover, we further confirmed the above findings with miR-10a-5p expression in validation cohort and GEPIA online database. As shown in Figure S1A-C, significant upregulation of miR-10a-5p was also observed in primary BCa tissues and patients with MIBC. Plasma miR-10a-5p expression level was significantly higher in the MIBC group compared to the NMIBC or HC group $(P<$ 0.001, Figure S1D). The clinicopathological analysis showed that miR-10a-5p expression was significantly correlated with tumor grade, histological grade, and $\mathrm{T}$ stage in validation cohort (Table S1).

\section{The Diagnostic Value of miR-10a-5p in BCa Patients}

To evaluate the diagnostic value of miR-10a-5p in $\mathrm{BCa}$ patients, we analyzed the performance of plasma miR-10a-5p in distinguishing $\mathrm{BCa}$ patients from $\mathrm{HC}$ patients using $\mathrm{ROC}$ analysis in the training cohort. As shown in Figure 2A and Table 2, the optimal diagnostic cut-off value for miR-10a-5p was 1.09, and the AUC value for miR-10a-5p was 0.815 (95\% confidence interval [CI], 0.734-0.896), with a sensitivity and specificity of $79.5 \%$ and $65.6 \%$, respectively, in distinguishing the BCa patients from HC patients. Next, the ROC curve was used to explore the potential of utilizing miR-18a as a biomarker for differentiating MIBC from NMIBC. As 
Table I The Relationship Between miR-10a-5p Expression and Clinical Features in Patients with Bladder Cancer

\begin{tabular}{|c|c|c|c|c|}
\hline \multirow[t]{2}{*}{ Parameters } & \multirow{2}{*}{$\begin{array}{l}\text { Cases } \\
(n=88)\end{array}$} & \multicolumn{2}{|c|}{ miR-I0a-5p, n (\%) } & \multirow[t]{2}{*}{$P$-value } \\
\hline & & $\begin{array}{c}\text { High } \\
\text { Expression } \\
(n=44)\end{array}$ & $\begin{array}{c}\text { Low } \\
\text { Expression } \\
(n=44)\end{array}$ & \\
\hline Age, year & & & & 0.509 \\
\hline$<60$ & $33(37.5)$ & $18(40.9)$ & $15(34.1)$ & \\
\hline$\geq 60$ & $55(62.5)$ & $26(59.1)$ & $29(65.9)$ & \\
\hline Gender & & & & 0.325 \\
\hline Male & $66(75.0)$ & $35(79.5)$ & $31(70.5)$ & \\
\hline Female & $22(25.0)$ & $9(20.5)$ & $13(29.5)$ & \\
\hline Tumor grade & & & & $0.008^{*}$ \\
\hline NMIBC & $32(36.4)$ & $10(22.7)$ & $22(50.0)$ & \\
\hline MIBC & $56(63.6)$ & $34(77.3)$ & $22(50.0)$ & \\
\hline $\begin{array}{l}\text { Histological } \\
\text { grade }\end{array}$ & & & & $0.030^{*}$ \\
\hline Low & $36(40.9)$ & $13(29.5)$ & $23(52.3)$ & \\
\hline High & $52(59.1)$ & $31(70.5)$ & $21(47.7)$ & \\
\hline T stage & & & & $0.033^{*}$ \\
\hline Tis-TI & $42(47.7)$ & $16(36.4)$ & $26(59.1)$ & \\
\hline T2-T4 & $46(52.3)$ & $28(63.6)$ & $18(40.9)$ & \\
\hline $\begin{array}{l}\text { Lymph nodes } \\
\text { metastasis }\end{array}$ & & & & 0.334 \\
\hline Yes & II (I2.5) & $7(15.9)$ & $4(9.1)$ & \\
\hline No & $77(87.5)$ & $37(84.1)$ & $40(90.9)$ & \\
\hline Multiplicity & & & & 0.338 \\
\hline Single & $64(72.7)$ & $30(68.2)$ & $34(77.3)$ & \\
\hline Multiple & $24(27.3)$ & $14(3 \mid .8)$ & $10(22.7)$ & \\
\hline
\end{tabular}

Note: $* P<0.05$.

Abbreviations: MIBC, muscle invasive bladder cancer; NMIBC, non-muscle invasive bladder cancer.

shown in Figure 2B and Table 2, the optimal diagnostic cut-off value for miR-10a-5p was 1.28 , and the AUC value for miR$10 \mathrm{a}-5 \mathrm{p}$ was 0.785 (95\% CI, 0.688-0.883), with a sensitivity and specificity of $75.0 \%$ and $64.2 \%$, respectively, in distinguishing the MIBC patients from NMIBC patients. Subsequently, the diagnostic power of miR-10a-5p was confirmed in the validation cohort. As demonstrated in Figure S2, miR-10a-5p still had the great value to distinguish $\mathrm{BCa}$ patients form $\mathrm{HC}$ patients with the AUC of 0.954 (0.688-0.883), and further to differentiate MIBC patients from NMIBC patients with the AUC of $0.796(0.688-0.883)$.

\section{The Prognostic Value of miR-10a-5p in BCa Patients}

Next, by generating Kaplan-Meier curves of OS, our results showed that $\mathrm{BCa}$ patients with a high level of
miR-10a-5p was associated with worse OS (Figure 3A; $P=0.005$ ) and RFS (Figure $3 \mathrm{~B} ; P=0.002$ ) compared with patients with a low level of miR-10a-5p in the training cohort. By using univariate and multivariate $\mathrm{COX}$ regression analysis (Tables 3 and 4), we found that tumor grade (HR: 2.02, 95\% CI: 1.32-2.85, $P=0.028)$, T stage (HR: 1.81, 95\% CI: $1.51-2.13, P=0.001)$, and high miR-10a-5p expression (HR: 1.74, 95\% CI: 1.31-2.01, $P=0.002$ ) were independent indicators of poor OS in $\mathrm{BCa}$ patients; and tumor grade (HR: 2.52, 95\% CI: 1.16-3.31, $P=0.035$ ), T stage (HR: 2.11, 95\% CI: 1.21-3.05, $P=0.001$ ), and high miR-10a-5p expression (HR: 1.95, 95\% CI: 1.20-2.64, $P=$ 0.001 ) were independent indicators of poor RFS in $\mathrm{BCa}$ patients. Additionally, upregulation of miR-10a-5p correlated with suboptimal OS (Figure S3A; $P=0.008$ ) and RFS (Figure $\mathrm{S} 3 \mathrm{~B} ; P=0.016$ ) in the validation cohort. The COX regression analysis (Tables $\mathrm{S} 2$ and $\underline{\mathrm{S} 3}$ ) further verified the prognostic role miR-10a-5p for $\mathrm{BCa}$ in the validation cohort. Taken together, our data indicated that miR-10a$5 \mathrm{p}$ is an independent favorable prognostic factor in $\mathrm{BCa}$ patients.

\section{Relationship Between miR-I0a-5p and the Proliferation, Migration, and Invasion Ability of BCa Cells}

Next, we further investigated the proliferation, migration, and invasion abilities of miR-10a-5p in BCa tumor progression using CCK-8 and Transwell assays. Firstly, we overexpressed and knocked down the expression level of miR-10a-5p in $253 \mathrm{j}$ cells using miR-10a-5p mimics and inhibitors $(P<0.001$, Figure 4A). The CCK-8 assay results showed that the proliferation of the $253 \mathrm{j}$ cell lines transfected with the miR-10a-5p mimics was significantly increased compared with that of the cells transfected with the negative control, while the proliferation of the $253 \mathrm{j}$ cell lines transfected with the miR-10a-5p inhibitors was significantly inhibited (Figure 4B). Transwell assays demonstrated that miR-10a-5p mimics led to a significant increase in the migratory and invasive capability of 253 j cells $(P<0.001$, Figure $4 C)$, whereas miR$10 \mathrm{a}-5 \mathrm{p}$ inhibitors markedly suppressed the migration and invasion of $253 \mathrm{j}$ cells compared to that in respective controls $(P<0.001$, Figure 4D). In addition, as revealed in Figure 5, the promoting role of miR-10a-5p overexpression as regards cell proliferation, migration, and invasion was confirmed in another $\mathrm{BCa}$ cell lines $\mathrm{J} 82$. 
A

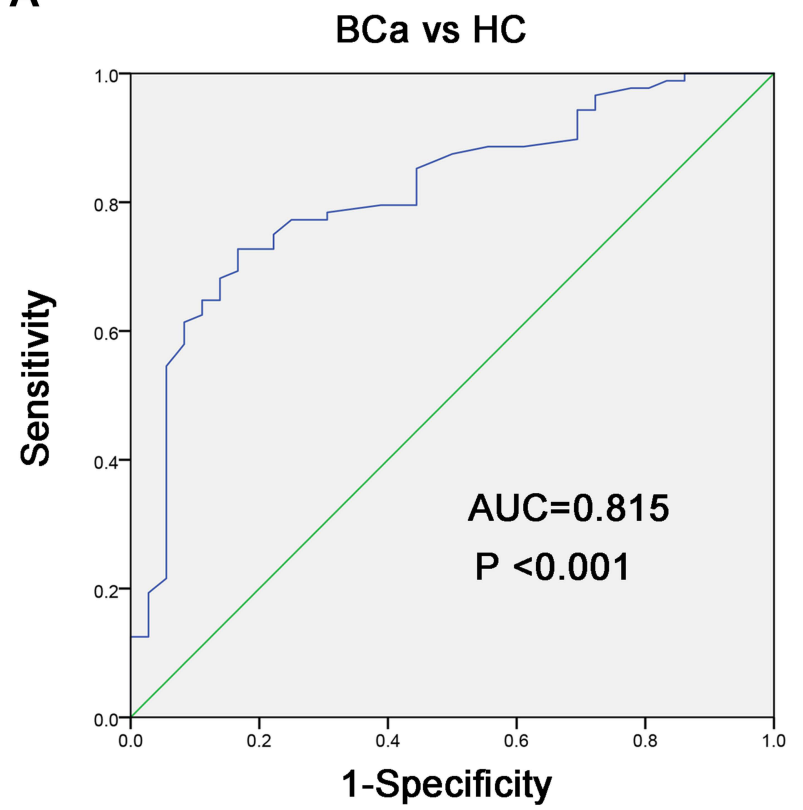

B

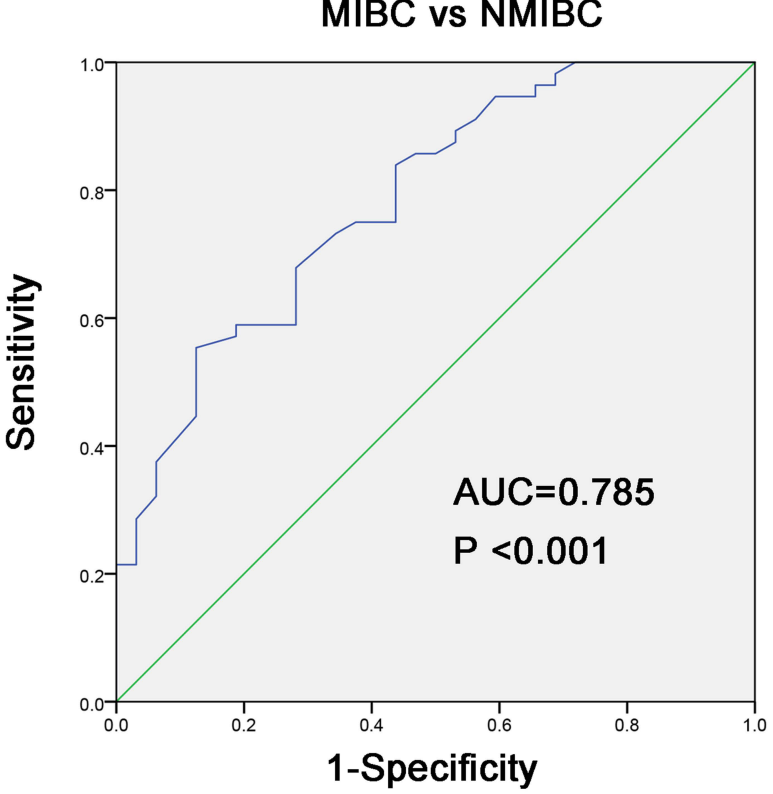

Figure 2 Diagnostic value assessment of miR-10a-5p in bladder cancer. (A) ROC curves indicate the ability of plasma miR-10a-5p to distinguish BCa patients from HC patients. (B) ROC curves indicate the ability of plasma miR-I0a-5p to distinguish MIBC patients from NMIBC patients.

Abbreviations: MIBC, muscle invasive bladder cancer; NMIBC, non-muscle invasive bladder cancer.

\section{Discussion}

Currently, a great number of studies have shown that miRNAs are abnormally expressed and may play crucial roles in diagnostic, prognostic, or biological functions in BCa progression. For instance, Wataru et al reported a miRNA panel, including miR-6087, miR-6724-5p, miR-3960, miR-1343-5p, miR1185-1-3p, miR-6831-5p and miR-4695-5p, which could discriminate bladder cancer from healthy volunteers. ${ }^{24}$ Moreover, Lin et al established a predictive model to predict the overall survival of bladder cancer based on 7 miRNAs including miR185-5p, miR-663a, miR-30c-5p, miR-3648, miR-1270, miR200c-3p, and miR-29c-5p. ${ }^{25}$

Various studies have implicated miR-10a-5p in tumor progression. For example, Takayuki et al revealed the antitumor role of miR-10a-5p in renal cell carcinoma that low expression of miR-10a-5p was correlated to worse overall survival and overexpression of miR-10a-5p could inhibit cancer cell migration and invasion. ${ }^{21}$ Moreover, Zhai et al reported that downregulation of miR-10-5p inhibited the cell viability and promoted cell cycle arrest of cervical cancer cells. ${ }^{22}$ In addition, Xiong et al determined that miR-10a-5p was an independent adverse prognostic factor in patients with pancreatic ductal adenocarcinoma (PDAC) and promoted progression of PDAC cells in vitro and vivo. ${ }^{17}$ In this study, through analyzing the clinical samples, we found that miR-10a-3p was upregulated in $\mathrm{BCa}$ tissues and further increased in patient with MIBC compared to those with NMIBC both in tumor samples and plasma samples. Our study also revealed the close relationship between miR-10a$5 \mathrm{p}$ expression and advanced tumor grade, histological grade, and $\mathrm{T}$ stage. High expression of miR-10a-5p closely correlated with advanced clinicopathological characteristics and poor survival outcomes.

Up to date, cystectomy is still the most reliable and accessible method for the detection of bladder cancer, which is expensive, invasive, time-consuming, and cannot be widely used especially for developing countries. ${ }^{26}$ With the development of microarray technology, a great number

Table 2 ROC Curves Analysis for the Diagnostic Power of Plasma miR-10a-5p in BCa

\begin{tabular}{|l|l|l|l|l|l|}
\hline Group & AUC (95\% Confidence Interval) & P value & Cut-Off Value & Sensitivity & Specificity \\
\hline BCa vs HC & $0.815(0.734-0.896)$ & $<0.001$ & 1.09 & 79.5 & 65.6 \\
MIBC vs NMIBC & $0.785(0.688-0.883)$ & $<0.001$ & 1.28 & 75.0 & 64.2 \\
\hline
\end{tabular}

Abbreviations: $\mathrm{BCa}$, bladder cancer; MIBC, muscle invasive bladder cancer; NMIBC, non-muscle invasive bladder cancer. 
A

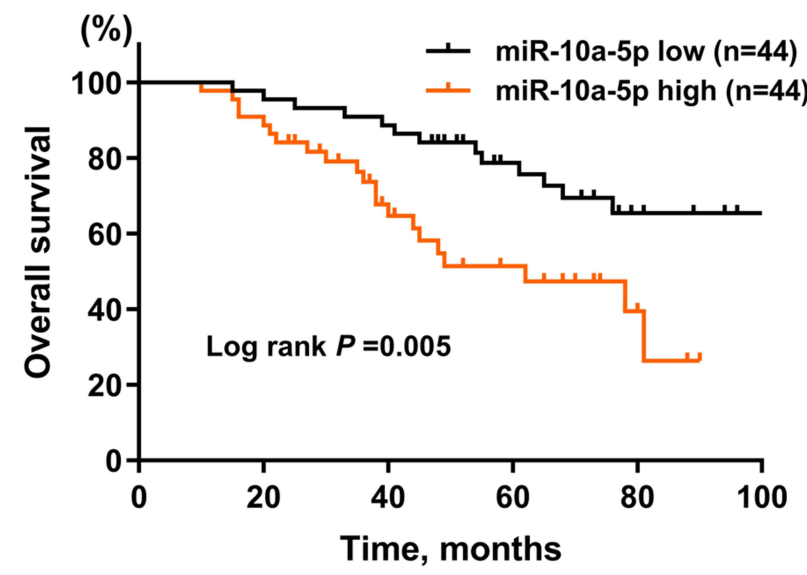

B

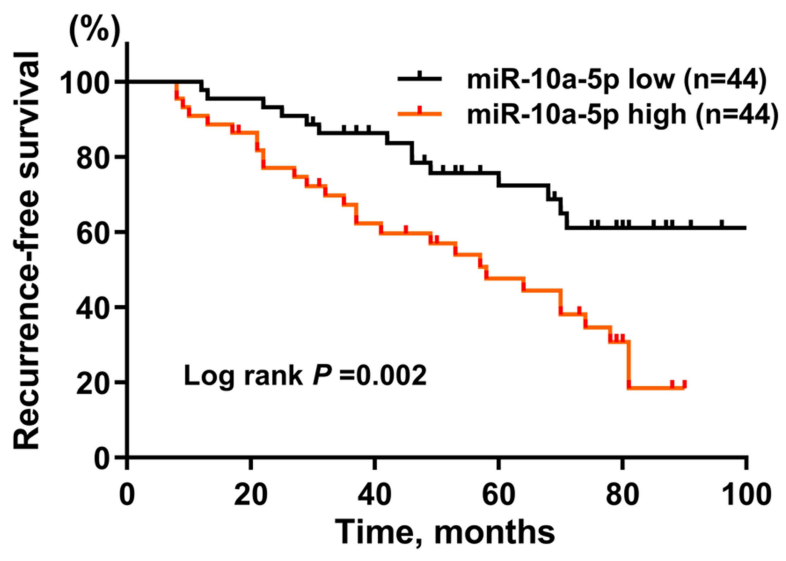

Figure 3 Kaplan-Meier curves of overall survival $(\mathbf{A})$ and recurrence-free survival $(\mathbf{B})$ for $B C a$ patients stratified by miR-I0a-5p expression in $B C a$ tissues. Abbreviation: $\mathrm{BCa}$, bladder cancer.

of biomarkers, including miRNAs, have been developed for the early diagnosis of multiple cancers. ${ }^{27}$ Specific serum miRNAs are very stable in blood plasma and serum, which is more convenient and noninvasive to predict the initiation and progression of $\mathrm{BCa} .{ }^{28}$ As reported in acute myeloid leukemia (AML), miR-10a-5p had

Table 3 Univariate and Multivariate Analyses of Prognostic Factors Associated with Overall Survival

\begin{tabular}{|c|c|c|c|c|}
\hline \multirow[t]{2}{*}{ Variables } & \multicolumn{2}{|l|}{ Univariate } & \multicolumn{2}{|l|}{ Multivariate } \\
\hline & HR (95\% CI) & $P$-value & HR (95\% Cl) & $P$-value \\
\hline Age ( $\geq 60$ vs $<60$ ) & $1.09(0.74-1.63)$ & 0.154 & & \\
\hline Gender (male vs female) & I.04 (0.7I-I.72) & 0.395 & & \\
\hline Tumor grade (MIBC vs NMIBC) & $2.13(1.54-2.96)$ & $0.003^{*}$ & $2.02(1.32-2.85)$ & $0.028^{*}$ \\
\hline Histological grade (high vs low) & $1.56(0.89-2.35)$ & 0.097 & & \\
\hline T stage (T2-T4 vs Tis-TI) & $1.98(1.64-2.32)$ & $<0.00 I^{*}$ & $1.8 \mathrm{I}(1.5 \mathrm{I}-2.13)$ & $0.001 *$ \\
\hline Lymph nodes metastasis (yes vs no) & I.I5 (0.72-I.86) & 0.111 & & \\
\hline Multiplicity (high vs low) & $1.29(0.8 \mathrm{I}-\mathrm{I} .84)$ & 0.136 & & \\
\hline miR-10a-5p expression (high vs low) & $1.85(1.46-2.15)$ & $0.001 *$ & $1.74(1.31-2.01)$ & $0.002 *$ \\
\hline
\end{tabular}

Note: $* p<0.05$

Abbreviations: MIBC, muscle invasive bladder cancer; NMIBC, non-muscle invasive bladder cancer; HR, hazard ratio; $\mathrm{Cl}$, confidence interval.

Table 4 Univariate and Multivariate Analyses of Prognostic Factors Associated with Recurrence-Free Survival

\begin{tabular}{|c|c|c|c|c|}
\hline \multirow[t]{2}{*}{ Variables } & \multicolumn{2}{|l|}{ Univariate } & \multicolumn{2}{|l|}{ Multivariate } \\
\hline & HR (95\% CI) & $P$-value & HR (95\% Cl) & $P$-value \\
\hline Age $(\geq 60$ vs $<60)$ & $1.16(0.8 \mathrm{I}-1.75)$ & 0.263 & & \\
\hline Gender (male vs female) & I.II (0.68-1.65) & 0.185 & & \\
\hline Tumor grade (MIBC vs NMIBC) & $2.85(1.76-3.94)$ & $0.00 I^{*}$ & $2.52(1.16-3.31)$ & $0.035 *$ \\
\hline Histological grade (high vs low) & $1.31(0.76-2.12)$ & 0.194 & & \\
\hline T stage (T2-T4 vs Tis-TI) & $2.64(1.32-3.42)$ & $<0.00 I^{*}$ & $2.11(1.2 I-3.05)$ & $0.001 *$ \\
\hline Lymph nodes metastasis (yes vs no) & $1.32(0.65-1.98)$ & 0.351 & & \\
\hline Multiplicity (high vs low) & I.I $9(0.91-1.45)$ & 0.656 & & \\
\hline miR-10a-5p expression (high vs low) & $2.08(1.35-2.95)$ & $<0.00 I^{*}$ & $1.95(1.20-2.64)$ & $0.001 *$ \\
\hline
\end{tabular}

Note: $* P<0.05$

Abbreviations: MIBC, muscle invasive bladder cancer; NMIBC, non-muscle invasive bladder cancer; HR, hazard ratio; $\mathrm{Cl}$, confidence interval. 
A

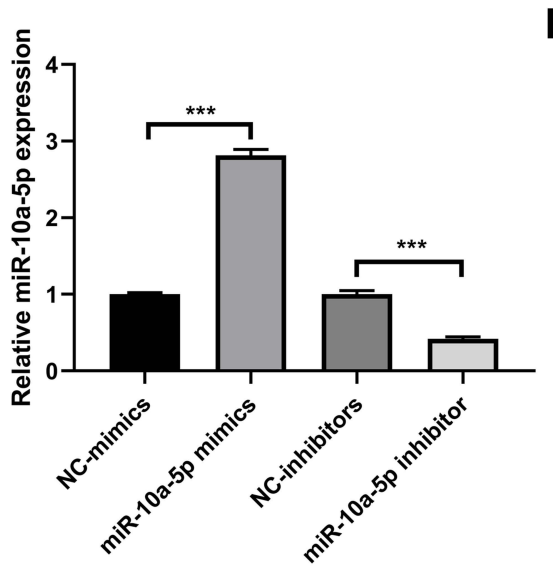

C

D
B

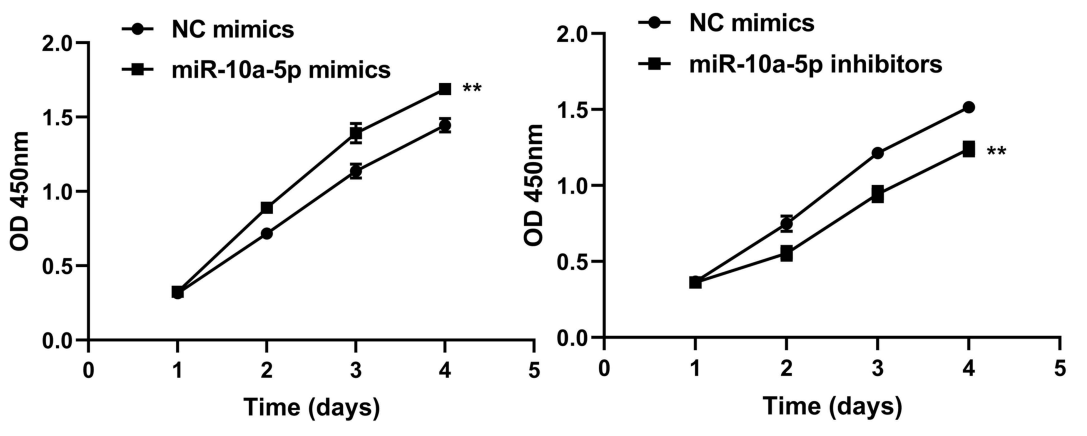

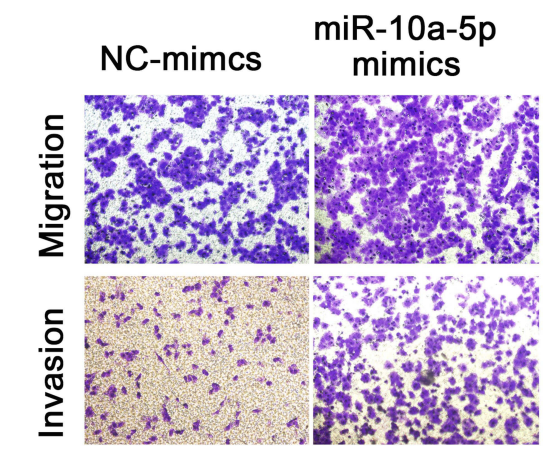

miR-10a-5p
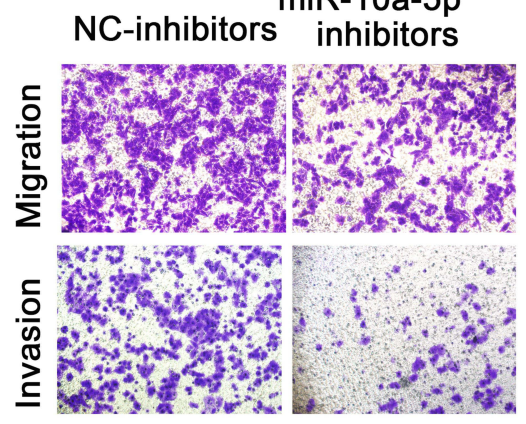
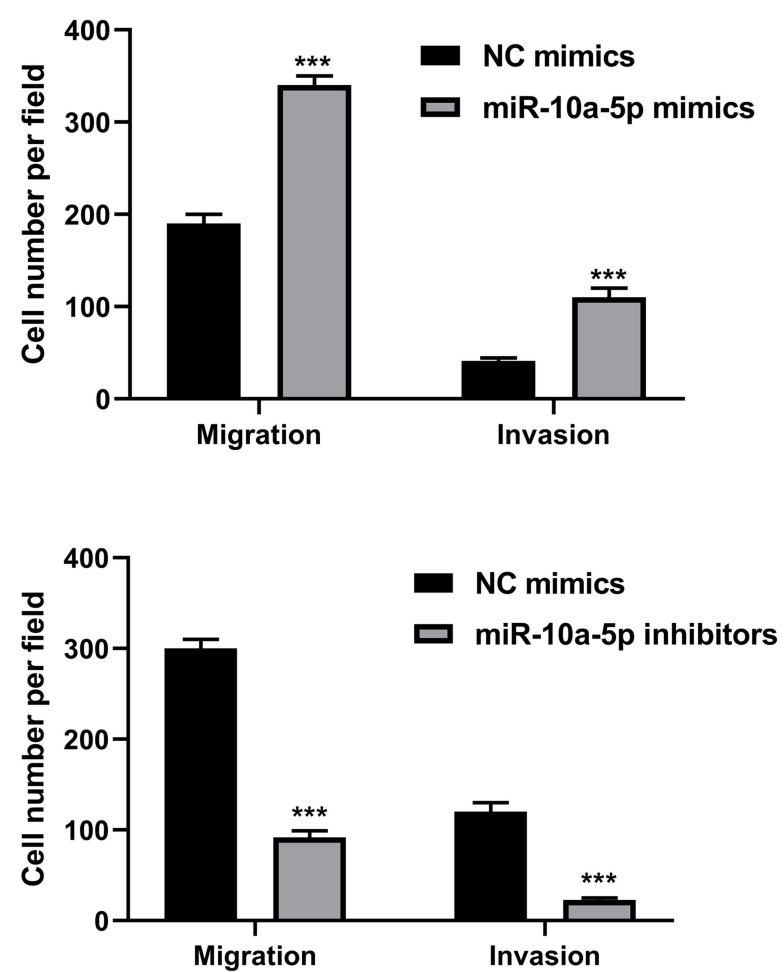

Figure 4 MiR-10a-5p promotes BCa cell proliferation, migration, and invasion. (A) RT-qPCR analysis of miR-10a-5p expression levels in 253j cells after transfection with miR-10a-5p mimics/inhibitors and respective negative controls. (B) CCK8 assays were performed to test the effect of miR-10a-5p mimics or inhibitors on cell proliferation of $253 \mathrm{j}$ cells. (C) Transwell assays were performed to test the effect of miR-10a-5p mimics on cell migration and invasion of $253 \mathrm{j}$ cells (magnification: $200 \times$ ). (D) Transwell assays were performed to test the effect of miR-10a-5p inhibitors on cell migration and invasion of $253 \mathrm{j}$ cells (magnification: $200 \times$ ). $* * P<0.0 \mathrm{I}, * * * P<0.00 \mathrm{I}$.

Abbreviation: $\mathrm{BCa}$, bladder cancer.

important diagnostic value in differentiating AML from normal subjects. ${ }^{18}$ In addition, Bao et al showed that miR$10 \mathrm{a}-5 \mathrm{p}$ was upregulated in non-small cell lung cancer (NSCLC) and also had great value for the clinical diagnosis of patients with NSCLC. ${ }^{20}$ However, the diagnostic and biological role of miR-10a-5p have not been elucidated in BCa. In this study, our results indicated that circulating plasma miR-10a-5p expression had the great performance power to discriminate $\mathrm{BCa}$ patients form $\mathrm{HC}$ patients before surgery, and further to differentiate MIBC from NMIBC. In addition, our biological function assays determined the promoting role of miR-10a-5p in cell proliferation, migration, and invasion of $\mathrm{BCa}$ cells. However, this study had its limitation that the underlying mechanism of miR-10a-5p biological function is unclear.

In conclusion, our results demonstrate that miR-10a-5p was upregulated in BCa patients, and miR-10a-5p could act as a promising diagnostic and prognostic biomarker for $\mathrm{BCa}$ patients. Moreover, miR-10a-5p exerted a tumor promoting role during the $\mathrm{BCa}$ cell progression. 
A

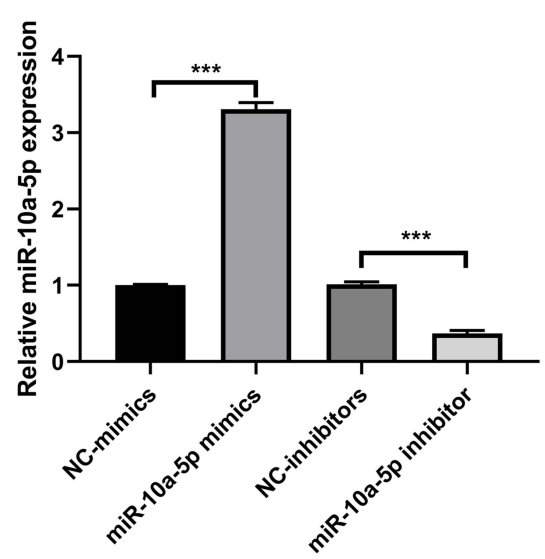

C

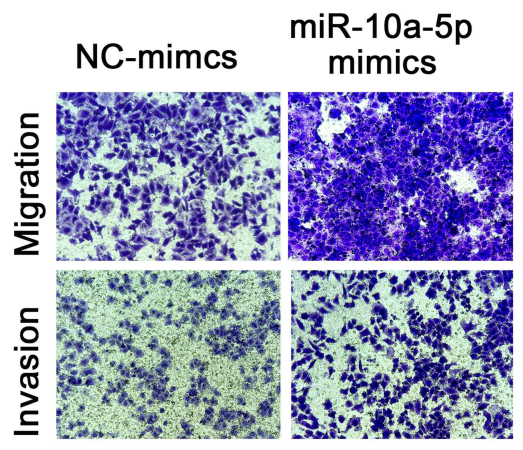

miR-10a-5p

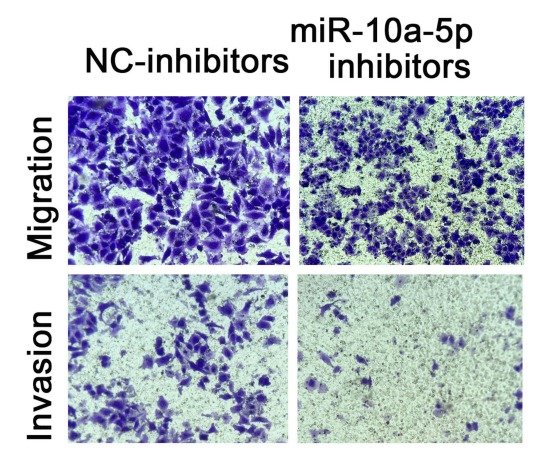

D
B
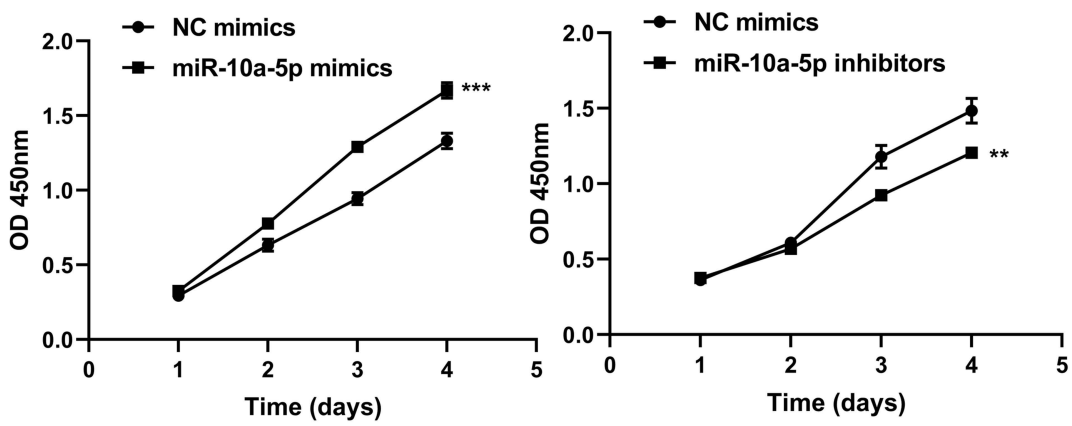

Figure 5 MiR-10a-5p promotes BCa cell proliferation, migration, and invasion. (A) RT-qPCR analysis of miR-10a-5p expression levels in J82 cells after transfection with miR$10 a-5 p$ mimics/inhibitors and respective negative controls. (B) CCK8 assays were performed to test the effect of miR-10a-5p mimics or inhibitors on cell proliferation of 382 cells. (C) Transwell assays were performed to test the effect of miR-10a-5p mimics on cell migration and invasion of J82 cells (magnification: $200 \times$ ). (D) Transwell assays were performed to test the effect of miR-10a-5p inhibitors on cell migration and invasion of J82 cells (magnification: $200 \times$ ). $* * P<0.01$, $* * * P<0.00 \mathrm{I}$.

Abbreviation: $\mathrm{BCa}$, bladder cancer.

\section{Data Sharing Statement}

All data generated in this study will be made available on reasonable request.

\section{Funding}

There is no funding to report.

\section{Disclosure}

The authors report no conflicts of interest in this work.
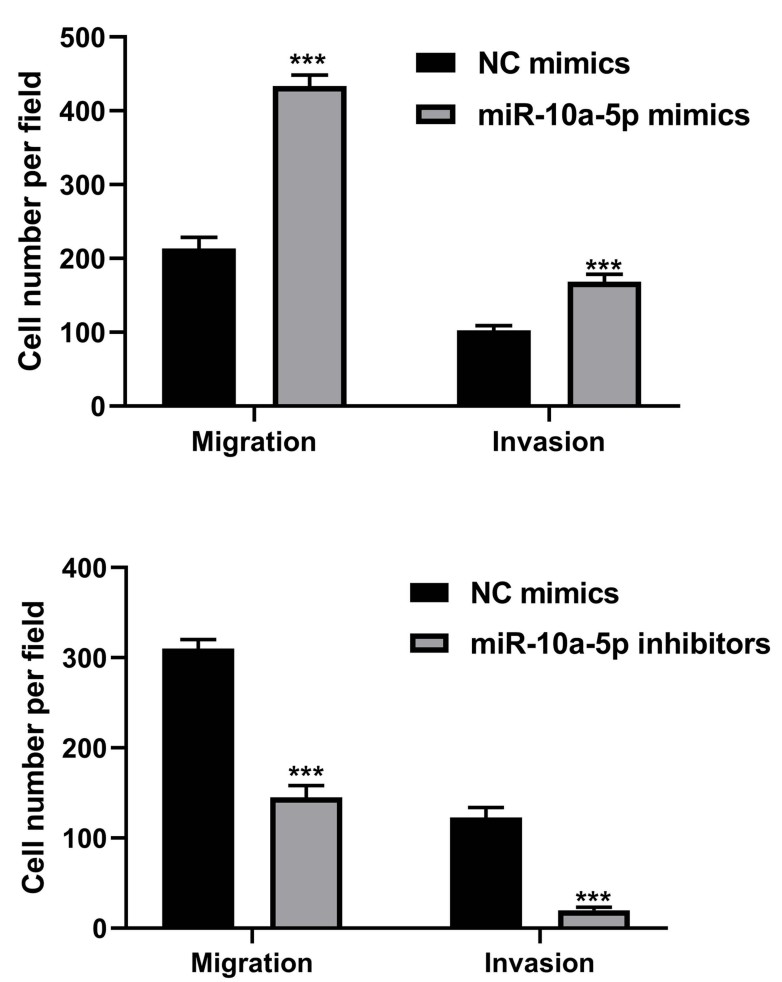
4. Moschini M, D’Andrea D, Korn S, et al. Characteristics and clinical significance of histological variants of bladder cancer. Nat Rev Urol. 2017;14(11):651-668. doi:10.1038/nrurol.2017.125

5. DeGeorge KC, Holt HR, Hodges SC. Bladder Cancer: diagnosis and Treatment. Am Fam Physician. 2017;96(8):507-514.

6. Fankhauser CD, Mostafid H. Prevention of bladder cancer incidence and recurrence: nutrition and lifestyle. Curr Opin Urol. 2018;28 (1):88-92. doi:10.1097/MOU.0000000000000452

7. McConkey DJ, Choi W. Molecular Subtypes of Bladder Cancer. Curr Oncol Rep. 2018;20(10):77. doi:10.1007/s11912-018-0727-5

8. Felsenstein KM, Theodorescu D. Precision medicine for urothelial bladder cancer: update on tumour genomics and immunotherapy. Nat Rev Urol. 2018;15(2):92-111. doi:10.1038/nrurol.2017.179

9. Sweis RF, Galsky MD. Emerging role of immunotherapy in urothelial carcinoma-Immunobiology/biomarkers. Urol Oncol. 2016;34 (12):556-565. doi:10.1016/j.urolonc.2016.10.006

10. Martinez Rodriguez RH, Buisan Rueda O, Ibarz L. Bladder cancer: present and future. Med Clin (Barc). 2017;149(10):449-455. doi:10.1016/j.medcli.2017.06.009

11. Tutar Y. miRNA and cancer; computational and experimental approaches. Curr Pharm Biotechnol. 2014;15(5):429. doi:10.2174/ 138920101505140828161335

12. Qadir MI, Faheem A. miRNA: a Diagnostic and Therapeutic Tool for Pancreatic Cancer. Crit Rev Eukaryot Gene Expr. 2017;27 (3):197-204. doi:10.1615/CritRevEukaryotGeneExpr.2017019494

13. Sun Z, Shi K, Yang S, et al. Effect of exosomal miRNA on cancer biology and clinical applications. Mol Cancer. 2018;17(1):147. doi:10.1186/s12943-018-0897-7

14. Barbato S, Solaini G, Fabbri M. MicroRNAs in Oncogenesis and Tumor Suppression. Int Rev Cell Mol Biol. 2017;333:229-268.

15. Wieczorek E, Reszka E. mRNA, microRNA and lncRNA as novel bladder tumor markers. Clin Chim Acta. 2018;477:141-153. doi:10.1016/j.cca.2017.12.009

16. Cochetti G, Vermandois JA, Maulà V, et al. Role of miRNAs in prostate cancer: do we really know everything? Urol Oncol. 2020;38(7):623-635. doi:10.1016/j.urolonc.2020.03.007

17. Xiong G, Huang H, Feng M, et al. MiR-10a-5p targets TFAP2C to promote gemcitabine resistance in pancreatic ductal adenocarcinoma. J Exp Clin Cancer Res. 2018;37(1):76. doi:10.1186/s13046-0180739-x
18. Zhi Y, Xie X, Wang R, et al. Serum level of miR-10-5p as a prognostic biomarker for acute myeloid leukemia. Int J Hematol. 2015;102(3):296-303. doi:10.1007/s12185-015-1829-6

19. Guo L, Li Y, Zhao C, et al. RECQL4, Negatively Regulated by miR-10a-5p, Facilitates Cell Proliferation and Invasion via MAFB in Ovarian Cancer. Front Oncol. 2020;10:524128. doi:10.3389/ fonc. 2020.524128

20. Bao M, Pan S, Yang W, Chen S, Shan Y, Shi H. Serum miR-10a-5p and miR-196a-5p as non-invasive biomarkers in non-small cell lung cancer. Int J Clin Exp Pathol. 2018;11(2):773-780.

21. Arai T, Okato A, Kojima S, et al. Regulation of spindle and kinetochore-associated protein 1 by antitumor miR-10a-5p in renal cell carcinoma. Cancer Sci. 2017;108(10):2088-2101. doi:10.1111/ cas. 13331

22. Zhai L, Li Y, Lan X, Ai L. MicroRNA-10a-5p suppresses cancer proliferation and division in human cervical cancer by targeting BDNF. Exp Ther Med. 2017;14(6):6147-6151.

23. Livak KJ, Schmittgen TD. Analysis of relative gene expression data using real-time quantitative PCR and the 2(-Delta Delta $\mathrm{C}(\mathrm{T})$ ) Method. Methods. 2001;25(4):402-408. doi:10.1006/meth.2001.1262

24. Usuba W, Urabe F, Yamamoto Y, et al. Circulating miRNA panels for specific and early detection in bladder cancer. Cancer Sci. 2019;110 (1):408-419. doi:10.1111/cas.13856

25. Lin GB, Zhang CM, Chen XY, et al. Identification of circulating miRNAs as novel prognostic biomarkers for bladder cancer. Mathematical Biosci Eng. 2019;17(1):834-844. doi:10.3934/ mbe. 2020044

26. Takata R, Katagiri T, Kanehira M, et al. Predicting response to methotrexate, vinblastine, doxorubicin, and cisplatin neoadjuvant chemotherapy for bladder cancers through genome-wide gene expression profiling. Clin Cancer Res. 2005;11(7):2625-2636. doi:10.1158/ 1078-0432.CCR-04-1988

27. Shi HB, Yu JX, Yu JX, et al. Diagnostic significance of microRNAs as novel biomarkers for bladder cancer: a meta-analysis of ten articles. World J Surg Oncol. 2017;15(1):147. doi:10.1186/s12957017-1201-9

28. Tölle A, Blobel CC, Jung K. Circulating miRNAs in blood and urine as diagnostic and prognostic biomarkers for bladder cancer: an update in 2017. Biomark Med. 2018;12(6):667-676. doi:10.2217/ bmm-2017-0392

\section{Publish your work in this journal}

Cancer Management and Research is an international, peer-reviewed open access journal focusing on cancer research and the optimal use of preventative and integrated treatment interventions to achieve improved outcomes, enhanced survival and quality of life for the cancer patient.
The manuscript management system is completely online and includes a very quick and fair peer-review system, which is all easy to use.

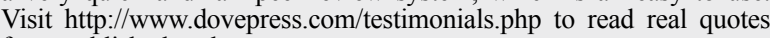
from published authors. 